\title{
Effects of five-minute mindfulness meditation on mental health care professionals
}

\begin{abstract}
This study aimed to examine the use of five-minute mindfulness meditation to reduce the stress level, in terms of perception of stress in one's life, and to increase the mindfulness level, in terms of the level of attentiveness to the present moment purposefully and without judgment, among mental health care professionals for seven consecutive days. In addition, the correlation between mindfulness level and stress level was examined. This quantitative research study utilized a pre- and post-test quasi-experimental design using Freiburg Mindfulness Inventory to measure mindfulness level and Perceived Stress Scale to measure stress level. 61 participants, who were active mental health care professionals at The Chicago School located in Chicago, IL, Irvine, CA and Los Angeles, CA, received seven days of five-minute mindfulness meditation exercise via the internet between November 2013 and January 2014. The medians from the pre- and post-tests of the intervention group showed a significant reduction $(p<.001)$ in stress level, while there was not a significant difference $(\mathrm{p}<.105)$ in mindfulness level. There was a significant inverse correlation $(\mathrm{p}$ $<.01)$ between mindfulness and stress. The results of this study suggest that five-minute mindfulness meditation could be an effective method in decreasing stress in this mental health care professional sample.
\end{abstract}

Keywords: mindfulness meditation, meditation, mindfulness, stress reduction, selfcare, mental health care professionals, psychologist, therapist, psychiatrist, five-minute meditation
Volume 2 Issue 3 - 2015

\author{
Amy G Lam, Sean Sterling, Edward Margines \\ The Chicago School of Professional Psychology, USA
}

Correspondence: Amy G Lam, 2540 I Cabot Rd, Ste. I I6,
Laguna Hills, CA 92653, USA, Tel 949-732-0323,

Email dramylam@gmail.com

Received: August 08, 2014 | Published: March 26, 2015

\section{Background of the problem}

Mental health care professionals often work long hours and in situations that are taxing and challenging. Workloads are increasing, as are family obligations. As helping professionals spend more time with clients, they are exposed to an increasing number of upsetting stories about suffering and trauma. This second-hand trauma is one of the major causes of burnout. In addition, the accumulated stress from clinical work is one of the major causes for emotional exhaustion, depression, and even suicides in working health care professionals. ${ }^{1}$ According to American Psychological Association, ${ }^{2}$ psychologists should be aware of the effect of their mental and physical statuses on their clinical performance. It is important to be aware of the potential hazards that mental health care professionals face and for them to be cognizant of the need for self-care as well as patient care. Self-care practice can help create a balanced life for health care professionals. Regular relaxation practice such as mindfulness meditation has been proven to provide many beneficial effects of reducing stress and anxiety level and increasing quality of life. ${ }^{3}$ In recent Western medicine, mindfulness-based meditation has been shown to successfully treat many stress-related diseases. ${ }^{4}$ According to Ledesma \& Kumano, ${ }^{5}$ major stress, that causes depression and anxiety in patients, may result from the diagnosis of a chronic illness. Conversely, stress is also one of the causes of physical and psychological illnesses. However, mindfulness meditation requires considerable time and consistency. Vettese et al., ${ }^{6}$ reported less than one-quarter of their meditation participants followed through with the daily 30 - to 60 -minute mindfulness home practice, and nearly half failed to demonstrate the positive outcomes link to the practice. Thus, a shorter but effective version of mindfulness meditation than the original 1.5 hour meditation would be useful for many. The mindfulness-based interventions or meditations in the literature are at minimum 1.5 hour long with 45 -minute home practices. There has been no research to date on the impacts of five-minute mindfulness meditation.

\section{Relationship between well-being and mindfulness meditation}

According to Wallace and Shapiro, ${ }^{7}$ well-being "is a way of engaging with life based on a wholesome way of life, mental balance, and a sound understanding of reality" (p. 698-699). People lose their balances, become ill or have disease, because of non-agreeable "sensory, behavioral, intellectual, or aesthetic stimuli". ${ }^{7}$ However, according to Buddhism, people can train their minds to correct these imbalances through meditation. ${ }^{8}$

Most people function in a very automatic and habitual way most of the time as how Brown \& Ryan' described people being "mindless and less awake states" (p.823). It is in complete opposite of the way of mindfulness about being mindful and aware of the present moment. Such mindfulness is important in changing the automatic and habitual way of feeling, thinking and behaving as it facilitates self-regulation. According to Deci \& Ryan, ${ }^{10}$ and Dalai Lama XIV, ${ }^{11}$ mindfulness meditation trains one's mind to be open to awareness. As soon as one is aware at the present moment, the here-and-now, change of the self can occur. Mindfulness meditation involves cognitive attention which brings the hidden habitual subjects, including behavior, emotion and thought, to the conscious mind. Once these hidden subjects become conscious, the individual then has an option to change any unpleasant behavior, emotion and thought, as well as to confront and accept them, as mindfulness meditation also involves perceiving the present stimuli without any judgment by seeing them as they are. This mindful awareness and attention without any judgment initiate the healthenhancing changes. ${ }^{9}, 12$ According to Brown \& Ryan, ${ }^{9}$ there is a strong link between well-being and mindfulness meditation, as mindfulness meditation trains one's mind to be aware to the present moment where the change of oneself can occur. 
According to Kabat-Zinn, ${ }^{13}$ mindfulness-based interventions reduce stress and depression in chronic pain patients. Similarly, Shapiro et al., ${ }^{14}$ in their study on the effects of mindfulness-based stress reduction on medical students, showed significant decrease in anxiety and depression. Shapiro et al., ${ }^{15}$ showed beneficial results from two-hour mindfulness-based stress reduction on health care professionals. However, according to Vettese et al., ${ }^{6}$ and Shapiro et al., ${ }^{15}$ some of the participants dropped out due to the time-demanding nature of mindfulness meditation and increase of responsibility. Health care professionals usually have a demanding schedule with insufficient time for relaxation or mental breaks. ${ }^{15}$ Clearly, health care professionals, who are actively involved in clinical work, are in need of a beneficial and time-efficient method to release their stress and improve their life satisfaction, which may also enhance their work performance.

\section{Research questions}

With high stress levels and demanding schedules among mental health care professionals, the research questions are as follow:

I. Will five-minutes of mindfulness meditation once per day for one week decrease the level of stress among mental health care professionals?

II. Will five-minutes of mindfulness meditation once per day for one week increase the level of mindfulness among mental health care professionals?

III. Is there a clinically significant inverse relationship between mindfulness and stress?

\section{Research design and methodology}

The design of this study used a quasi-experimental research method to perform a pre-post comparison of participants' level of stress and mindfulness in addition to comparing the post-test results between mindfulness level and stress level. A quantitative method was utilized with two reliable and validated inventories, which were the 14-item Freiburg Mindfulness Inventory ${ }^{16-18}$ and the 10 -item Perceived Stress Scale. ${ }^{19}$

\section{Participants}

Participants were recruited from The Chicago School of Professional Psychology and its counseling centers via The Chicago School's email system. All of the participants of this study were active mental health care professionals, including active mental health therapist, psychological intern or trainee, psychoanalyst, psychologist, psychiatrist, or mental health counselor, of all gender, ethnicity and religious background with age 21 or older and were literate in English language. Participants were selected through a screening procedure using a 2-item demographic questionnaire.

\section{Kinds of measurements for all variables}

Mindfulness level was measured by the Freiburg Mindfulness Scale, while stress level was measured by the Perceived Stress Scale. The Perceived Stress Scale might create reactivity in participants, as it may influence the outcome of the Freiburg Mindfulness Scale. Although participants were not being observed during the experiment, the Perceived Stress Scale might bring back some stressful emotions for the participants. Therefore, participants were asked to take the Freiburg Mindfulness Scale prior to taking the Perceived Stress Scale to minimize the reactivity.
The Freiburg Mindfulness Inventory is a valid and reliable 14item scale, which uses a 4-point Likert scale with 1 as "rarely" to 4 as "almost always". The inventory captures several aspects of mindfulness and does not require the knowledge of the background of Buddhist mindfulness. It was developed in mindfulness retreats for experienced practitioners to assess the here-and-now experience without any judgment and the openness to negative experience. ${ }^{16-18}$ According to Walach et al., ${ }^{18}$ internal reliability of the Freiburg Mindfulness Inventory has a coefficients alpha of 0.86 .

The Perceived Stress Scale is a 10 -item scale to measure the degree of one's current situations, which perceives as stressful. The scale includes direct questions that measure the levels of experienced stress. It takes a few minutes to complete the Perceived Stress Scale with its scores being obtained by reversing responses to the four positively stated items and then summing across all scale items. The questions are relatively free of content targeting any minority group and are of general nature about current feelings and thoughts. ${ }^{19}$ The internal reliability of the Perceived Stress Scale ranged from alpha 0.84 to 0.86. It was validated in two student samples and a clinical sample. ${ }^{19}$ The predictive validity in studies where the evidence of higher Perceived Stress Scale scores were associated with more colds, ${ }^{20}$ more prone to depressive symptoms due to stressful life events ${ }^{21}$ and failure to control blood sugar levels in diabetics. ${ }^{22}$

\section{Procedures}

The sample for this study was one group receiving five-minute mindfulness meditation exercises. Participants were recruited via emails, and the sample was a convenience sample. The study was conducted online, and participants were first asked to complete the electronic consent before proceeding to the 2-item demographic questionnaire and the 24-item pre-test survey, which included the Freiburg Mindfulness Inventory and the Perceived Stress Scale. Upon completing the pre-test survey, participants were redirected to the meditation page, where the instructions of the mindfulness meditation exercises and the five-minute mindfulness meditation audio file were located. Participants were asked to bookmark the meditation page for reference use throughout the participation duration. Participants were instructed to practice the five-minute mindfulness meditation once a day at any convenient location and time of the day, except during driving or operating machinery equipment, for seven consecutive days, beginning from the same day that participants completed the pre-test survey. Upon completion of the seventh day of mindfulness meditation exercises, all participants were asked to complete the post-test survey online. Participants were instructed to click on a link, which redirected participants to the 24-item post-test survey, on the meditation page. The post-test survey included the Freiburg Mindfulness Inventory and the Perceived Stress Scale. Data were then collected via internet using a free online survey tool, Survey Gizmo. The statistical tests that were utilized in this study was one-sample median test to assess the pre-post test scores of the independent variable and Spearman Correlation to measure the relationship between the two dependent variables. Median test scores were used for comparison due to the type of measurement that was utilized to measure the dependent variable was ordinal.

\section{Results}

\section{Hypothesis I}

Participants' stress levels were measured by the Perceived Stress Scale prior to and after the week of exercises. Using a one-sample median test to compare the pre- and post-test scores of the Perceived 
Stress Scale, the results shown in Table 1 indicated that there was a reduction in the stress level of the Intervention Group after a week of five-minute mindfulness meditation exercises. The stress scores between the pre- and post-tests were significantly different at the $p<$ .05 level. The obtained $p$-value was less than .001 .

Table I Levels of Stress Before and After Exposure to a Week of Five-minute Mindfulness Meditation

\begin{tabular}{lll}
\hline Test & N & Median \\
\hline Pre & 61 & 21 \\
Post & 30 & $9.5^{*}$ \\
\hline
\end{tabular}

*Statistical significance $(p<.05)$

\section{Hypothesis 2}

The mindfulness scores of the Intervention Group was collected prior to and after participating in a week of five-minute mindfulness meditation exercises as measured by the Freiburg Mindfulness Inventory. Using one-sample median test for each sample, the preand post-test mindfulness scores were compared. As observed in Table 2, the exercises did not produce a significant increase in the mindfulness level of the Intervention Group. The results indicated that the two groups of scores were not significantly different at the $p<.05$ level. The obtained p-value of .105 approached significance, however.

Table 2 Levels of Mindfulness Before and After Exposure to a Week of Fiveminute Mindfulness Meditation

\begin{tabular}{lll} 
Test & N & Median \\
\hline Pre & 61 & 36 \\
Post & 30 & 46 \\
\hline
\end{tabular}

\section{Hypothesis 3}

An inverse correlation between mindfulness and stress as measured by the Freiburg Mindfulness Inventory, and the Perceived Stress Scale $_{2}$ was hypothesized. Using Spearman Correlation to compute the relationship between post test scores of the Freiburg Mindfulness Inventory and the Perceived Stress Scale from the Intervention Group $(\mathrm{n}=30)$, it was determined that mindfulness is inversely correlated to stress. The result indicated that the inverse correlation between two variables, mindfulness and stress, was significant at the $p<.05$ level. Among participants who completed in the entire study, the Spearman's rho was -.51 with the obtained $p$-value less than .01 .

\section{Discussion}

\section{Hypothesis I}

From the findings of this study, stress level did significantly decrease after a week of five-minute mindfulness meditation exercise among mental health care professionals. After participating in this study, participants reported significant decreases in the followings:

a. being upset because of something that happened unexpectedly,

b. unable to control the important things in life,

c. feeling nervous and stressed,

d. not able to cope with all the things that participant had to do,

e. being angered by things that were outside of participant's control, and

f. feeling difficulties were piling up so high that participant could not overcome them.
Ledesma \& Kumano ${ }^{5}$ comparatively reported that cancer patients, who participated in the traditional one-hour mindfulness-based therapy showed positive improvement on anxiety and stress. These cancer patients became more accepting of self and less reactive to stress. Similar to Ledesma \& Kimano, ${ }^{5}$ Tacon et al., ${ }^{23}$ found a significant decrease in stress and anxiety on women with breast cancer after being administered with mindfulness-based therapy.

Mindfulness-based meditation has shown positive results in individuals with mental health problems, such as social anxiety disorder ${ }^{24}$ and PTSD. ${ }^{25}$ Similarly, Goldin \& Gross $^{26}$ and McManus et al., ${ }^{27}$ argued that patients with social anxiety disorder showed a decrease in negative emotional experience after the application of mindfulness-based meditation, while Williams et al., ${ }^{28}$ commented the mindfulness-based therapy reduced anger and depression in suicidal individuals. Brown \& Ryan ${ }^{9}$ found that there is a correlation between emotional intelligence and mindfulness meditation. Their study reported that individuals who practice mindfulness meditation are less likely to react negatively to stressful situations and are more tranquil. Similarly, couples who practice mindfulness meditation have less mood disturbance and negative reactions to stressful events. ${ }^{29}$ Shapiro et al., ${ }^{15}$ study suggested that mindfulness-based stress reduction (MBSR) reduced stress level on health care professionals and reported significant decrease of stress level and anxiety level on therapists in training after attending MBSR class. ${ }^{3}$ According to Tacon et al., ${ }^{23}$ MBSR significantly reduced anxiety and stress on women with breast cancer and decreased negative mood states during the post-treatment. Similarly, McManus et al. ${ }^{27}$ reported that mindfulness-based intervention decreased anxiety level and increased self-acceptance and the ability to relax in individuals with social anxiety disorder.

\section{Hypothesis 2}

It was hypothesized that mindfulness level will increase significantly from a week of five-minute mindfulness meditation exercises among mental health care professionals. The obtained p-value of 105 did not show a significant increase. This non-significant finding might have been a result of some participants having little to no meditation experience prior to this study. According to Namto, ${ }^{30}$ novice meditators may experience a great degree of inner turmoil mentally, while Goldstein \& Kornfield ${ }^{31}$ argued that novice meditators may become apathetic due to their resistance to the changes and experiences brought on from the meditation. This indicated that it is possible that novice meditators may not have developed mindfulness in comparison to experienced meditators. Since this study did not categorized participants by their level of meditation experience, the increased mindfulness level of the more experienced meditators might have been obscured among the novice meditators. According to Boorstein $^{32}$ and Goleman, ${ }^{33}$ attention and awareness to emotions and thoughts in communication may be enhanced through mindfulness meditation, while Shapiro et al., ${ }^{34}$ argued that individuals who practice mindfulness meditation are more attentive to their emotions and thoughts without judgment. Lykins \& Baer $^{35}$ has conducted a study on mindfulness meditation comparing between non-meditators and experienced meditators. The findings of their study showed an increase in self-compassion, self-regulation and mindfulness level among the experienced meditators.

Another possible explanation for the insignificant increase of mindfulness level in this study may be due to the morphological changes in the brain. According to Grant et al., ${ }^{36}$ and Lazar et al., ${ }^{37}$ experienced meditators are trained to focus their attention to an internal or external stimulus, for example, the breath or a bodily 
position, which is controlled by the somatotopic area of the brain. Morphological changes may occur after a long period of such training. Similarly, experienced meditators were found to have thicker cortex and more gray matter in the pain-related regions of the brain when compared to non-meditators. This difference indicated increase of awareness, decrease in pain sensitivity and improved control of emotions in the experienced meditators than the non-meditators. ${ }^{36,38}$ In addition, Goldin \& Gross $^{26}$ has found a decrease in the amygdalal activity which is responsible for processing and expression of emotions and an increase in the brain network related to attention regulation among patients with social anxiety disorder after being exposed to eight weeks of MBSR.

\section{Hypothesis 3}

According to the statistical findings of this study, stress is significantly inversely correlated with mindfulness, which was hypothesized and expected. Findings were generated from the posttest results from both the Freiburg Mindfulness Inventory and the Perceived Stress Scale.

Mindfulness meditation evolved from a practice of mindful concentration on a topic, which was discovered some 2500 years ago by an Indian man named Shakyamuni Buddha while in search of the answers for the worldly suffering. ${ }^{39}$ Mindfulness meditation operates as a form of self-healing by releasing one from suffering through an altered state of mind.$^{40}$ Dr. Jon Kabat-Zinn was a pioneer in studying the effects of mindfulness-based meditation and the inventor of MBSR. His scientific studies showed reduction of stress and anxiety in couples ${ }^{29}$ and health care professionals after a period of exposure to MBSR. Additionally, Kabat-Zinn successfully treated individuals with stress-related diseases with MBSR, which suggested that mindfulness is inversely correlated to stress. ${ }^{41}$ According to Fairfax, ${ }^{42}$ mindfulness is being integrated into intervention models such as Mindfulness-based Cognitive Therapy (MBCT), ${ }^{27,28,43} \mathrm{MBSR}^{29}$ and Mindfulness-based Relapse Prevention (MBRP) ${ }^{25}$ because of the high success rate in reducing stress and treating other symptoms by using mindfulness-based interventions. According to Vujanovic et al., ${ }^{25}$ mindfulness-based therapy lowered anxiety and traumarelated symptoms in patients with PTSD, while Hofmann et al., ${ }^{43}$ and $\mathrm{Niazi}^{44}$ reported that mindfulness-based therapy reduced anxiety and depression in patients with mood disorders. These findings were further supported by Tacon et al., ${ }^{23}$ and Ledesma \& Kumano ${ }^{5}$ whose studies found that cancer patients became less stressful and less reactive to stress after being exposed to mindfulness-based therapy. Similarly, individuals who practice mindfulness meditation are found less inclined to react negatively to stressful situations. ${ }^{9} \mathrm{Niazi}^{43}$ found that mindfulness meditation lessened negative reactions to stress and increased life-appreciation in both male and female cancer patients and diabetic patients.

\section{Implications of the findings}

In this study, five-minute mindfulness meditation had a significant beneficial impact. This suggested that mental health care professionals may meditate as little as five minutes a day to decrease their stress level and possibly to enhance their well-being if practiced consistently over a period of time. ${ }^{9,41}$ It also showed a significant reduction in stress in participants. This suggested that five-minute mindfulness meditation can have similar effects in cancer patients, and people with PTSD, social anxiety disorder, depression, anger issue, relational problems or suicidal ideations as the traditional 2-hour mindfulness meditation has. Mindfulness level did not significantly increase among mental health care professionals in this study but produced a potential trend toward increase. This indicated that extended application of fiveminute mindfulness meditation may increase mental health care professionals' mindfulness level. With increased mindfulness level, mental health care professionals may experience less stress or less inclination to react negatively to stressful situations and experience a decrease in anxiety, depression and burnout that may be caused by their therapeutic works., ${ }^{3,15}$ In addition, this finding also implied that mental health care professionals might enhance their abilities to provide a more therapeutic environment to their clients as they increase their self-compassion and positive feelings. ${ }^{3}$ Empathy and attentional qualities, ${ }^{40}$ and ability to work with stressful situations ${ }^{41}$ may also increase as mindfulness level increases with consistent practice of five-minute mindfulness meditation. In summary, the findings of this study suggested that mental health care professionals may enhance their qualities of life and work performance simply by meditating consistently with mindfulness-based meditation for five minutes a day.

\section{Limitations \& problems}

A limitation of this study is the length of the study. This study was executed for seven consecutive days, which may not have provided sufficient time for the mindfulness meditation to apply its full impact on the participants. Another limitation of this study is that the participants were self-selected to participate in this study, and have met the given criteria, including being a mental health care professional, age 21 or older and English-literate. These participants may have already come with an expectation of gaining some beneficial experiences through this meditation study. Because of this, there may be a significant difference between those who chose to participate and the ones who did not. Therefore, the findings of this study may only be pertinent to those who participated or would be willing to participate in this study but not to the general population. In addition, this study began with 61 volunteered participants but only 30 remained and completed the whole study. This raised an important question of why those who dropped out of the study chose to do so. One potential reason for this attrition is that those particular participants may have found the five minute mindfulness meditation exercises unpleasant or unhelpful. If that was the case, and those participants had managed to complete the study, their post-test scores would likely have reduced the significance of the obtained findings. Due to participant attrition, the findings based on the sample of this study that took part in this entire study may not be valid for the whole population of mental health care professionals. In addition, the results of this study may have very little generalizability to the general population due to the targeted population studied.

Participants were not categorized by their meditation experiences in this study. The results of mindfulness level may have been different if participants were categorized, because the experienced meditators may have already acquired a level of mindfulness than the novice meditators.

This study was conducted online without supervision, and participants may or may not have completed the study as instructed. In addition, both the Freiburg Mindfulness Inventory and the Perceived Stress Scale are self-reported measurements, which may not be as accurate because they are vulnerable to biased responding. These limitations result in lower validity of the findings.

\section{Recommendations for future research}

Further research is needed to understand the effects of fiveminute mindfulness meditation on mental health care professionals. 
It is recommended to categorize participants by their experiences of mindfulness meditation to avoid overlooking the differences of mindfulness levels between the novice and experienced meditators.

Another recommendation is extending the length of time of the study. This study was implemented for seven consecutive days. The full impact of the five-minute mindfulness meditation may continue to develop for longer than such a short interval. Thus, extending the time length might generate different results.

While future research can still be administered online, it is advisable to create a more structured procedure by providing a specific time each day for the participants to $\log$ online to practice the meditation together simultaneously. This method would provide the supervision that was missing in this study and could ensure that participants are following the timeline. In addition, this may decrease the dropout rate by enhancing the level of commitment from the participants. In future research, it would be advisable to request that all participants to fill out pre- and post-tests even if they chose to stop participating in the mindfulness meditation.

Furthermore, it would be relevant to further investigate the factors that differentiate those who adhere to the meditation schedule from those who chose not to do so. Another recommendation is to use physiological measurement, such as measuring cortisol levels, prior to and after the experiment in addition to the self-reported measures to assess changes in ongoing stress associated with participation in mindfulness meditation exercises.

\section{Acknowledgments}

None.

\section{Conflicts of interest}

Author declares there are no conflicts of interest.

\section{Funding}

None.

\section{References}

1. Spickard A, Gabbe SG, Christensen JF. Mid-career burnout in generalist and specialist physicians. JAMA. 2002;288(12):1447-1450.

2. American Psychological Association. Ethical principles of psychologists and code of conduct. Am Psychol. 2002;57(12):1060-1073.

3. Shapiro SL, Brown SW, Biegel GM. Teaching self-care to caregivers: Effects of mindfulness-based stress reduction on the mental health of therapists in training. Training and Education in Professional Psychology. 2007;1(2):105-115.

4. Schmit DT. The mesmerists inquire about "oriental mind power": West meets East in the search for the universal trance. J Hist Behav Sci. 2010;46(1):1-26.

5. Ledesma D, Kumano H. Mindfulness-based stress reduction and cancer: A meta-analysis. PsychoOncology. 2009;18(6):571-579.

6. Vettese LC, Toneatto T, Stea JN, et al. Do mindfulness meditation participants do their homework? And does it make a difference? A review of the empirical evidence. Journal of Cognitive Psychotherapy: An International Quarterly. 2009;23(3):198-225.

7. Wallace BA, Shapiro SL Mental balance and well-being: Building bridges between Buddhism and Western psychology. Am Psychol. 2006;61(7):690-701.

8. Dhamma R. The first discourse of the Buddha: Turning the wheel of Dharma. MA Wisdom, Boston, USA. 1997.
9. Brown KW, Ryan RM. The benefits of being present: Mindfulness and its role in psychological well-being. J Pers Soc Psychol. $2003 ; 84(4): 822-848$.

10. Deci EL, Ryan RM. Self-determination theory: When mind mediates behavior. Journal of Mind and Behavior. 1980;1(1):33-43.

11. Dalai Lama XIV. An open heart: Practicing compassion in everyday life. Little, Brown and Company, New York, USA. 2001. p.208.

12. Kabat-Zinn J. Full catastrophe living: Using the wisdom of your mind and body to face stress, pain, and illness. Delacorte, New York, USA. 1990.

13. Kabat-Zinn J. An outpatient program in behavioral medicine for chronic pain patients based on the practice of mindfulness meditation: Theoretical considerations and preliminary results. Gen Hosp Psychiatry. 1982;4(1):33-47.

14. Shapiro S, Schwartz GE, Bonner G. Effects of mindfulness-based stress reduction on medical and premedical students. J Behav Med. 1998;21(6):581-599.

15. Shapiro SL, Astin JA, Bishop SR, et al. Mindfulness-based stress reduction for health care professionals: Results from a randomized trial International Journal of Stress Management. 2005;12(2):164-176.

16. Baer RA, Smith GT, Hopkins J, et al. Using self-report assessment methods to explore facets of mindfulness. Assessment. 2006;13(1):27-45.

17. Kohls N, Sauer S, Walach H. Facets of mindfulness-results of an online study investigating the Freiburg mindfulness inventory. Personality and Individual Differences . 2009;46(2):224-230.

18. Walach H, Buchheld N, Buttenmuller V, et al. Measuring mindfulness: The Freiburg mindfulness inventory (FMI). Personality and Individual Differences. 2006;40(8):1543-1555.

19. Cohen S, Kamarck T, Mermelstein R. A global measure of perceived stress. J Health Soc Behav. 1983;24(4):385-396.

20. Cohen S, Tyrrell DA, Smith AP. Negative life events, perceived stress, negative effect, and susceptibility to the common cold. J Pers Soc Psychol. 1993;64(1):131-140.

21. Carpenter LL, Tyrka AR, McDougle CJ, et al. Cerebrospinal fluid corticotropin-releasing factor and perceived early-life stress in depressed patients and healthy control participants. Neuropsychopharmacology. 2004;29(4):777-784.

22. Kramer JR, Ledolter J, Manos GN, et al. Stress and metabolic control in diabetes mellitus: Methodological issues and an illustrative analysis. Ann Behav Med. 2000;22(1):17-28.

23. Tacon AM, Caldera YM, Ronaghan C. Mindfulness-based stressed reduction in women with breast cancer. Families, Systems, \& Health. 2004;22(2):193-203.

24. Goldin PR, Ramel W, Gross J. Mindfulness meditation training and selfreferential processing in social anxiety disorder: Behavioral and neural effects. J Cogn Psychother. 2009;23(3):242-257.

25. Vujanovic AA, Niles B, Pietrefesa A, et al. Mindfulness in the treatment of posttraumatic stress disorder among military veterans. Professional Psychology: Research and Practice. 2011;42(1):24-31.

26. Goldin PR, Gross JJ. Effects of mindfulness-based stress reduction (MBSR) on emotion regulation in social anxiety disorder. Emotion. 2010;10(1):83-91.

27. McManus F, Muse K, Surawy C. Mindfulness-based cognitive therapy (MBCT) for sever health anxiety. Journal of Healthcare Counseling \& Psychotherapy. 2011;11(1):19-23.

28. Williams JM, Duggan DS, Crane C, et al. Mindfulness-based cognitive therapy for prevention of recurrence of suicidal behavior. J Clin Psychol. 2006;62(2):201-210. 
29. Kabat-Zinn J. Mindfulness meditation: Health benefits of an ancient Buddhist practice. In: Goleman D \& Garin J (Eds.), Mind/Body Medicine. Consumer Reports, Yonkers, New York, USA. 1993. p.257-276.

30. Namto A. Insight meditation. Amarin Printing Group Ltd., Bangkok, Thailand. 1989.

31. Goldstein J, Kornfield J. Seeking the heart of wisdom: The path of insight meditation. Shambhala Publications Inc., Boston, USA. 1987.

32. Boorstein S. Transpersonal psychotherapy. State University of New York Press, Albany, New York, USA. 1996.

33. Goleman D. Social intelligence: The new science of human relationships. Bantam, New York, USA. 2006

34. Shapiro SL, Carlson LE, Astin JA, et al. Mechanisms of mindfulness. $J$ Clin Psychol. 2006;62(3):373-386.

35. Lykins ELB, Baer RA. Psychological functioning in a sample of longterm practitioners of mindfulness meditation. Journal of Cognitive Psychotherapy: An International Quarterly. 2009;23(3):226-241.

36. Grant JA, Courtemanche J, Duerden EG, et al. Cortical thickness and pain sensitivity in Zen meditators. Emotion. 2010;10(1):43-53.

37. Lazar SW, Kerr CE, Wasserman RH, et al. Meditation experience is associated with increased cortical thickness. Neuro Report. 2005;16(17):1893-1897.
38. Cahn BR, Polich J. Meditation states and traits: EEG, ERP, and neuroimaging studies. Psychol Bull. 2006;132(2):180-211.

39. Buksbazen JD. Zen meditation in plain English. Wisdom Publications, Boston, USA. 2002.

40. Walsh R, Shapiro SL. The meeting of meditative disciplines and Western psychology: A mutually enriching dialogue. American Psychologist. 2006;61(3):227-239.

41. Kabat-Zinn J. Mindfulness-based interventions in context: Past, present, and future. Clinical Psychology: Science and Practice. 2003;10(2):144-156.

42. Fairfax H. The use of mindfulness in obsessive compulsive disorder: Suggestions for its application and integration in existing treatment. Clin Psychology and Psychotherapy. 2008;15(1):53-59.

43. Hofmann SG, Sawyer AT, Witt AA, et al. The effect of mindfulnessbased therapy on anxiety and depression: A meta-analytic review. $J$ Consult Clin Psychol. 2010;78(2):169-183.

44. Niazi AK, Niazi SK. Mindfulness-based stress reduction: A nonpharmacological approach for chronic illnesses. $N$ Am J Med Sci. 2011;3(1):20-23. 